\title{
Three Dimensional Field Recording in Archaeology: An Example from Gabii
}

Rachel Opitz, Center for Advanced Spatial Technologies (CAST)

Department of Anthropology, University of Arkansas

\section{Introduction}

In asking for contributions to this series of posts reflecting on 3D modeling in archaeology, Bill Caraher posed a series of questions, one of which was:

"What is the future of 3D modeling in archaeology? At present, the 3D image is useful for illustrating artifacts and - in some cases - presenting archaeological and architectural relationships, but it has yet to prove itself as an essential basis for analysis or as a robust medium for communicating robust archaeological description. Will 3D visualization become more than just another method for providing illustrations for archaeological arguments?"

I'd very much like to answer "yes" to the question posed above. I'm going to argue that 3D modeling and visualizations can be the grounds for the re-interpretation of essential archaeological evidence, stratigraphic sequences, in a way that goes beyond just providing illustrations for arguments and conclusions drawn from other evidence. To make this argument, I'll start by providing a bit of background about 3D field recording at Gabii - an irreverent history of our digital documentation method and how it evolved.

\section{D at Gabii}

In 2009 Prof. Nicola Terrenato, with the enthusiasm and support of the Project's other directors, decided to open a fairly large excavation area at Gabii. Among the Project's priorities was the development of field survey method that would let us work quickly, so we could make efficient progress excavating in a big urban area. This would allow us to ask and maybe answer some big questions like 'How did the urban fabric develop, change over time, and decay at Gabii?' In addition to a fast survey-to-GIS-to-printed plan strategy, as an experiment, we were going to try recording some of the more complex contexts using photomodeling, as we called it after the Eos 
PhotoModeler software. Photomodeling would allow us to rapidly produce orthophotos of complex contexts, thereby avoiding tedious stone by stone survey in the field (Fig. 1).

Luckily in 2009 we didn't find much in the way of complex architecture or stratigraphy. We modeled a few contexts in the course of the season, and they turned out well. We were confident enough in the technique's efficacy that when an orientalizing infant burial was uncovered on the last day on site (see Becker and Nowlin, 2011) 3D modeling was our primary means of recording the remains and we were only a little nervous (Fig. 2). In 2010, when we started uncovering an Imperial necropolis and the remains of two houses, we used Photomodeling (more formally known as Structure From Motion -SFM- , or Image Based Modeling -IBM-, or Digital Close Range Photogrammetry -DCRP-) to record all the structural and human remains. Photomodeling swiftly, and a conscious decision to fully implement a new approach to the site's documentation, transitioned from being an experiment to being a key part of our recording strategy. In 2011 the scale of the excavation grew considerably. As a result of this rapid were left with a substantial backlog for model processing at the end of the field season. The need to undertake substantial amounts of post-season processing was obviously undesirable. Photomodeling was intended to speed up and facilitate the excavation and documentation process, and not add to the delays in producing documentation ready for interpretation and write-ups which are typical of all too many excavation projects. Post-season research in 2011 led us to switch to Agisoft Photoscan for the 2012 field season. The faster processing times and ability to batch script in python helped us to complete the 2011 field season's documentation in one season and get back on top of the workflow. 2012 was also the year that we introduced Unity3D on site as a tool for building and sharing more complex scenes $3 \mathrm{D}$ models of multiple contexts. This browser based approach to sharing was considered an improvement over the use of Meshlab or ArcGIS, both of which, while fundamentally simple to use, require download and install of software vieuld be viewed an obstacle.

2012 was also the year that we switched to LP Archaeology's ARK system for our descriptive data management (http:/ /ark.lparchaeology.com/) This was a fortuitous convergence, as having a web-based data management system meant that linking from the Unity3D content and from GIS content to database entries was fairly simple. Some 2012 post-season work was 
devoted to promoting ARK and Unity3D within the group as a means of distributing and presenting the models. Notably, several members of the excavation staff kindly agreed to participate in an interpretive experiment, which I discuss below. All of this was good news, because 2013 brought us the largest, most complex collection of structural remains to date. We finished the season with a small, but manageable backlog.

In short, the field recording system fundamentally works, six hundred odd individual models later. We've gone from SFM as an experiment to SFM as normal. I think this is one of the more important things we can say about 3D field recording at Gabii. It's just part of the routine - a proven method (Fig. 3).

All of the excavators feel that the interpretive sketch is still essential (a point on which we are entirely in agreement) and sometimes worry that all the excitement surrounding SFM and the speed it encourages us to document at will end up de-emphasizing actually looking at the archaeology, reflecting on what you see, and interpreting it via drawing carefully and slowly. This is an important ongoing debate about field recording within the team. The technology encourages us to speed up so we can work at larger scales, an important shift, as we've said, that permits us to ask and attempt to respond to large-scale questions, but the archaeology usually demands that we slow down and think about what we're doing. The integration of digital technologies and 'close range remote sensing' into the daily excavation routine therefore represents a constant balancing and re-balancing of practice, speeding up where we can and slowing down when needed. It takes discipline on the part of the entire excavation team to not drift into a false sense of security based on the seeming completeness of SFM models. To their credit, area supervisors and excavators actively promote conscientious documentation and remain on guard against letting the technology overtake careful documentation and thinking and interpretation in the field. I don't expect this particular conversation to end any time soon, nor would I want it to end, as I think this kind of constant monitoring of the balance between speed and detail, between rapid documentation of basic data and taking the time to make thoughtful interpretations is good field archaeology.

Five years in, we're still adapting our approach as the nature of the archaeology we are encountering changes, but for us most of the debate has 
moved from 3D recording as a field method to the implications of the 3D documentation for publication, communication and interpretation. I've taken a not inconsiderable amount of space to talk about the development of our field recording system because I think in order to talk about the question which Bill posed, about the role of 3D modeling beyond illustration, as part of analysis or as a robust communication medium, it has to be everywhere, fully integrated into the normal recording practice of an excavation. If it's something being done experimentally, for only a few contexts, or in a limited area, if it's not part of the excavator's practice, then it really can't be fully part of the interpretive stage or the communication and publication stage. Equally, it can't just be in the hands of the project's specialists. It has to be used by the project's team at large (something only starting to happen for us now). The length of the narrative above reflects the incremental way in which new technologies and practices develop and become part of excavation methodology, something that happens through a sustained effort over an extended period.

\section{Critically Evaluating Digital Data via Peer Review}

In 2003 $3_{\curlywedge}$ we embarked on an NEH funded project, 21st c. Data, 21st c. Publications: 3D Data and Building the Peer Reviewer Community, in which we are trying to use 3D content as more than a tool for documentation (m-gabii. adsroot.itcs.umich.edul). This project focuses on developing a process for the peer reviewed publication of the kinds of digital 3D models and complex, interactive datasets that projects like ours are now producing using SFM and related 3D field recording technologies, and building a community of peer reviewers with a shared frame of reference for evaluating these publications. This project is bringing us, at last, close to engaging with Bill's question, "Will 3D visualization become more than just another method for providing illustrations for archaeological arguments? [Can 3D modeling] prove itself as an essential basis for analysis or as a robust medium for communicating robust archaeological description?" The peer review process is designed to, among other things, help authors strengthen their argument and clarify its presentation. As researchers and academics, we're trained to recognize good and bad writing, and to identify holes and weaknesses in a argument. We learn what makes a useful chart or illustration. But what does good, useful interactive 3D content look like? What are the qualities on which it should be assessed? To develop a framework for the critique and review of what is essentially mixed visual and written content, 
we have to think about the link between visualization and interpretation and ask ourselves: Is visualization illustration, or part of the interpretive process and a means of interrogating the data? This question represents, I think, the nub of Bill's broader question about illustration, description and archaeological argument. In responding to this question, I would argue that these models should be understood in the context of the discipline of information visualization, as part of a school of visual communication and argument ranging from John Tukey's writing on Exploratory Data Analysis (1977) through Nathan Yau's posts on flowingdata.com.

This perspective no doubt stems from my work as an archaeologist away from the Gabii Project, in which I specialize in airborne laserscanning (ALS) applications in archaeology, a field in which information visualization and visual interpretation are viewed as fundamental parts of the research process. Consequently, I'm predisposed to think that creating and engaging with visualizations is part of the process of interpretation and understanding the physical remains 'on the ground' and the archaeology at hand. It's why I take the process of publishing and critiquing the models so seriously. I'm going to lift a few paragraphs from a volume I recently co-edited with David Cowley (RCAHMS) about airborne laserscanning in archaeology, which I think can be transposed fairly directly onto my thinking about 3D modeling in excavation contexts (http://www.oxbowbooks. com/oxbow/interpreting-archaeological-topography.html).

"In particular, the last decade has seen an exponential growth in the use and awareness of ALS [substitute here 3D modeling] by archaeologists and cultural resource managers[...] The powerful images produced, all promised a brave new world. And so it is - a world of possibilities and challenges, both in ensuring appropriate, archaeologically reliable applications that inform us about the past, but also in developing practices that integrate the strengths of new possibilities in manipulation and interrogation of vast digital datasets with so-called 'traditional' skills of archaeological observation and interpretation."

Engagement with digital 3D data "highlight[s] the importance of combining 'field-craft' and observation with the powerful algorithms and visualisation techniques that dense and/or extensive 3D data demand if we are to do anything more than scratch the surface." 
"In all cases the integration of 3D data into archaeological practices promotes the use of ever more sophisticated modelling and visualisations, from the creation of virtual replicas for display in a physical or digital museum or dissemination over the internet, to virtual reality and immersive visualization projects. Throughout, while the primary aim of these products may be to communicate and engage with a wide audience, these approaches also have a vital role for the investigating archaeologist in supporting interpretation where the visualization and measurement of very small scale and subtle features is essential (e.g. tool marks or rock art), and to under-pin spatial analyses such as viewsheds and least cost-paths, and inclusion in interactive virtual reality models. Universally, it is the use of $3 \mathrm{D}$ data as an articulation of archaeological topography that lies at the heart of the processes."ᄉ

It was with all this very much in mind that I posed the question in a paper I gave at the 2013 Meeting of the SAA: Does working with the models actually change our interpretations of the stratigraphy and therefore of the archaeology? This question sits at the intersection of what we do in the field and what we do in publication and as critical readers, and is closely tied up with one of the questions posed to contributors of this volume.

\section{An Experiment}

To start exploring these questions of interpretation, Marcello Mogetta (Unilyn Evans (Wriversity of Michigan), all members of the Gabii team, agreed to participate in this experiment. They respectively an assistant director, area supervisor, and assistant area supervisor at Gabii, and so represent different levels of experience and perspectives on the archaeology being uncovered.

I gave them all an assignment:

1. Pick a stratigraphic sequence in which you're interested, preferably one in which you recall us having done some $3 \mathrm{D}$ modeling.

2. Write down in brief your interpretation of the sequence and generally how you understand it.

3. I'll put together a model of the sequence, which we'll go through and look at together. 
4. I'll leave the model with you. You can decide if you now want to reinterpret anything. Write down, or tell me about your re-interpretations or lack thereof and if you found the model at all useful.

Each chose a stratigraphic sequence, Troy picked a series of floor surfaces within a room in an archaic complex (Fig. 4), Marilyn picked another series of surfaces which intersected with a tomb, while Marcello chose to look at a series of surfaces which might or might not have been part of the road which delimits the block containing the archaic compound on its eastern side. Each initially formed their interpretation using the-documentation other than the 3D models.

Troy began by constructing the following stratigraphic sequence and interpretation:

"SU 3163 (Truncated N-S Wall covers accumulation SU 3165 (not photo modeled)). SU 3165 covers collapse layer 3168 (collapse of earlier wall). This collapse layer covers two patches of one yellow floor surface (SUs 3180 and 3181) and three patches of a separate yellow floor surface (SUs $3192,3193,3194)$ identified and differentiated by proximity and color. These two floor surfaces are, potentially, separate floor preparations of the archaic compound that continues across the later drainage channel (Area D Room 2). A light brown silty layer of accumulation (3202) runs beneath the floor patches 3180, 3192, 3194, 3193 and a separate grey accumulation layer (3182) runs beneath 3181. Neither 3181 nor 3202 were photo modeled. My general understanding of this sequence is that there are two patchy heavily disturbed levels of archaic floor surface associated with a wall. This wall collapsed (3168) and a later wall (3163) was built on top of this collapse on a different alignment using the debris."

After working through the model together (to get over any hiccups with the interface) and leaving it with Troy for a while, he followed up with this set of notes:

"The 3D model itself, I think, shows some grounds for reinterpretation of the relationship I originally spelled out. Looking at this model, it seems that these patches of floor are part of one flooring event, not two distinct surfaces as I originally wrote. While patchy, there seems to be a level of connectivity across the whole model with the variances in presence and elevation possibly related to the destruction/collapse levels sitting on top 
of these layers. This was not clear from either the interpretations on the SU sheets or from the photographs. It is easier to think of and view these isolated patches as a single floor with the model than through other means of reconstruction (drawings/photos/etc.). I think, for this specific sequence, the ability to simultaneously view the different patches was something that the $3 \mathrm{D}$ model provided that, outside of a brief period pre-excavation, it was difficult to reconstruct. Because these two patches $(3180 / 3181$ and $3192 / 3193 / 3194$ ) were excavated on different days (probably by different students and certainly while other things were taking place) the model gave me the ability to look back and think about this corner of in a more cohesive way."

Marilyn had chosen to look at another room from the same archaic complex, further to the north (Fig. 5). Her question was more of a general exploration, where Troy's had been fairly specific. She was hoping to better understand a complex sequence of deposits and structures. We explored the assembled model of the room together, not coming to any immediate conclusions. We did strike upon one idea, looking at a gap in an alignment of stones interpreted as a wall in the complex. This gap, we noted, lines up nicely with the central pillar in the room. While a doorway had been identified in the northern wall of this room in the field, looking at the model suggested the idea that there might be another entrance, through the western wall. Nothing concrete, but an idea to play with and think about further during the next season of excavation. Marilyn noted that the idea of a doorway seemed increasingly satisfactory, and that it is not something we would have noticed without the freedom of movement, and that little bit of distance you can get exploring a digital model.

Marcello interested himself in a later sequence, related to the establishment and use of the system of streets which structures the urban plan at Gabii from about the 5th c. B.C. (Fig. 6). He originally noted: "Below the thick deposit of silt that obliterated the entire block corresponding to Area D (SU 3004=3049), the excavators identified a layer (SU 3053) whose limits coincided with one of the roads of the orthogonal town-plan (road 2, between Area C and D). The excavators interpreted SU 3053, therefore, as road surface. The road surface covered a deposit (SU 3066) which filled a cut in the bedrock containing multiple burials (SU 3081; Tombs 41-42). The tomb is located immediately east of the precinct wall which delimited the archaic compound (SU 2219). The west niche of the tomb was dug 
under the wall, causing its collapse at a later stage. The stratigraphy seems to provide crucial evidence to understand the sequence of occupation and general phasing of the site, showing that the creation of the orthogonal layout postdates the burials, which can be in turn connected with the abandonment of the archaic building."

He had a whole series of questions about the sequence, which had emerged from previous seasons of excavation: "Is SU 3053 really a road surface? What is its spatial relationship (especially in terms of elevation) with the deposits that cover the rich infant burial T48, farther to the south (SU 3134=3165)? Are these road surfaces too? Probably not, because they have a stratigraphic relationship with a series of abandonment layers (SU 3129, $3128,3117,3115)$. But how are these abandonment layers different from SU 3053?"

After looking at the model, the initial interpretation of the stratigraphic sequence was revised. He noted: "The model proved very useful for the interpretation of the road sequence, especially SU 3134. The limits of this SU correspond to those of road 2, as can be reconstructed on the basis of SU 3053. The SU seems to represent a leveling layer for the creation of road 2 (in fact, it seals the fill of a $500 \mathrm{BCE}$ infant burial). As a consequence, the structural feature next to it (SU 3163) may represent a retaining wall for the road, not a feature relating to the archaic compound (though perhaps it was built repurposing the collapse of the precinct wall). In light of this, I would now suggest that SU 3128 is the original road surface which was packed on top of the leveling layers (in fact it also includes a concentration of pebble-like stones, perhaps the glareata?). The elevations are consistent with this reconstruction. On the other hand, I now doubt that there is a direct stratigraphic relationship between SU 3134 and SUs 3129, 3117 and 3115 (it seems to me that this depends on the fact that SU 3134 was initially considered as extending west of structure SU 3163.”

So in all three cases the interpretation of the stratigraphic sequence changed, in more or less significant ways, after incorporating the 3D models into the interpretive process. I would argue, based on this experiment, that if our understanding of stratigraphic sequences, those fundamental building blocks of the interpretation of excavation data, are being changed on the basis of working with 3D models, then we are already beyond 'illustrations for arguments", and I feel we can answer "yes", 3D modeling is 
in the process of proving itself to be an essential basis for analysis and a robust means of archaeological communication, argument and narrative.

\section{Interim conclusions}

Methodological development is messy, and the impacts of new technologies on actual practice are usually indirect and only emerge later. The personalities involved are important, because the obstacle isn't so much the technology itself but rather our motivation to use it and our default behaviors, the tools we reach for when sifting through archaeological evidence, and the interaction between those tools and our thought processes as researchers and readers. These ingrained practices, our field habits and our desk habits, don't evolve quickly. We talk about 3D field recording and SFM as new technologies. The ground on 3D field recording at a large scale was broken by people like Dominic Powlesland working at West Heslerton in the early 1980s (http:/ /www.landscaperesearchcentre.org/), and we've had large scale SFM-based recording going at Gabii since 2009. 'New' is relative. Continued reassessment of our practices is essential, as is a willingness to go out on technical and methodological limbs. 3D modeling will only

get through the 'experimental' phase of the process bereby it becomes, a tool used by the archaeological community at large for analysis and as a robust means of making an argument if we actually try and use it to do these things, publish or otherwise share the results and the process, and are willing for it to occasionally go wrong. As always, it will take time and effort for new methods to become fully integrated into our interpretive work, our writing, our reading, and our way of thinking. We continue to work within the Gabii and 21st c. Data, 21st c. Publication Projects to use 3D modeling to record and interpret excavation data, make archaeological arguments and communicate them well, and make these data become part of the "normal" archaeological record, embedded in the conversational cycle of of publication $\mathrm{L}_{2}$ review $\mathrm{Critique}$ and response.

\section{Works Cited}

Becker, Jeffrey A. and Nowlin, J. (2011). "Orientalizing Infant Burials from Gabii, Italy." BABESCH 86:27-39. 
Opitz, R. (2013). "Digital Transitions: Technologies for Archaeological Fieldwork, Publishing and Community Engagement." SAA 78th Annual Meeting, Honolulu, Hawaii, April 3-7, 2013.

Tukey, John Wilder (1977). Exploratory Data Analysis. Reading, MA: Addison-Wesley. 

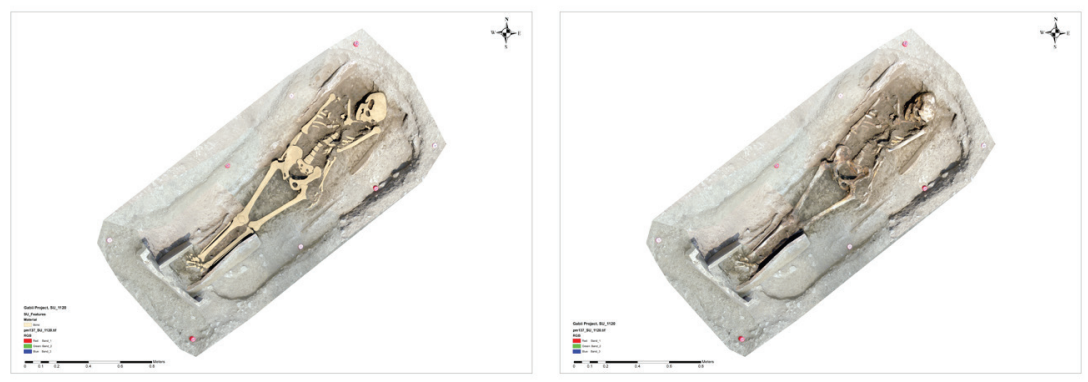

Figure 1: Detailed drawing using orthophotos generated from 3D models

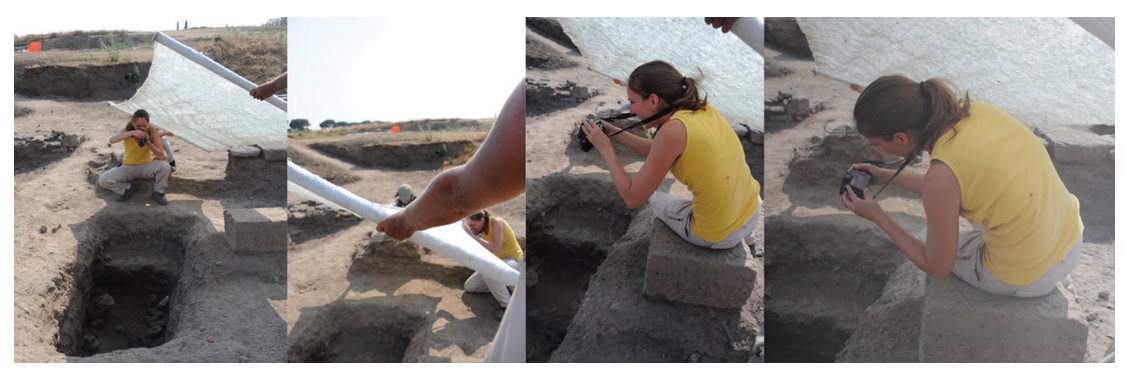

Figure 2: Jessica Nowlin taking photos for 3D model creation 


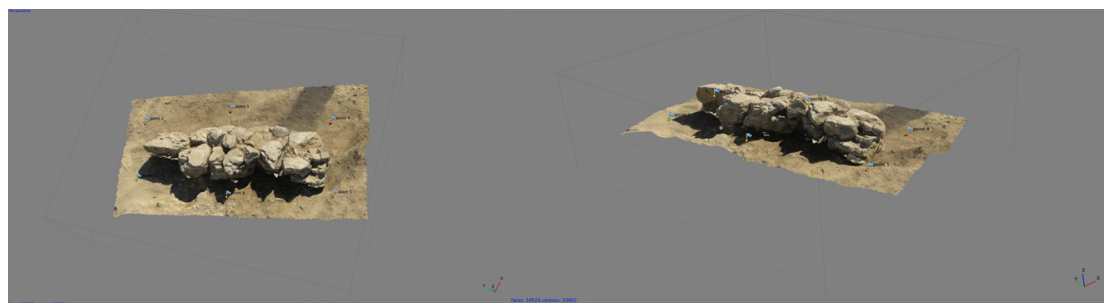

Figure 3: A typical single context model viewed in plan (left) and perspective

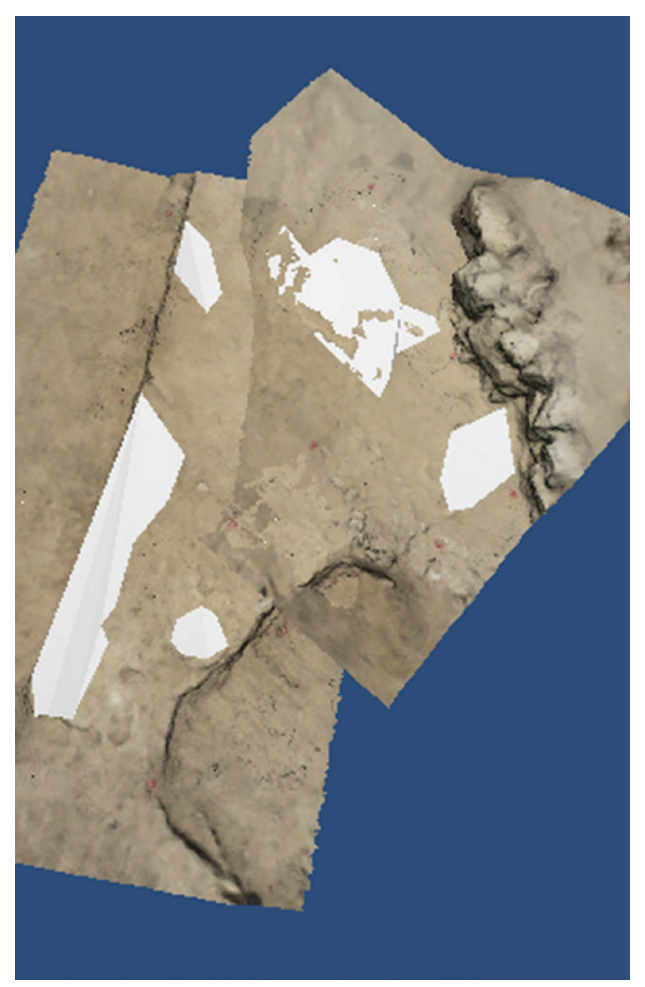

Figure 4: A screenshot of the scene put together to respond to Troy's question. White polygons indicate the locations of the surfaces in which we were interested. 


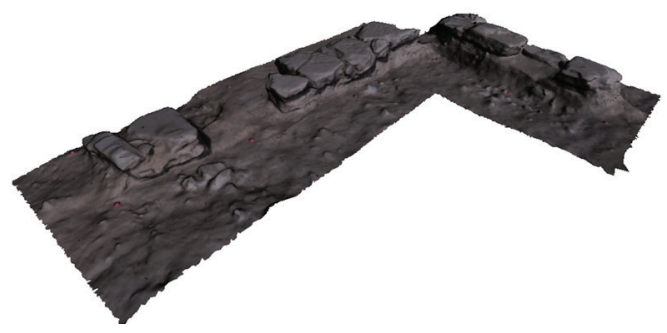

Figure 5: Two walls from the sequence Marilyn wanted to study, showing the gap in the west wall.

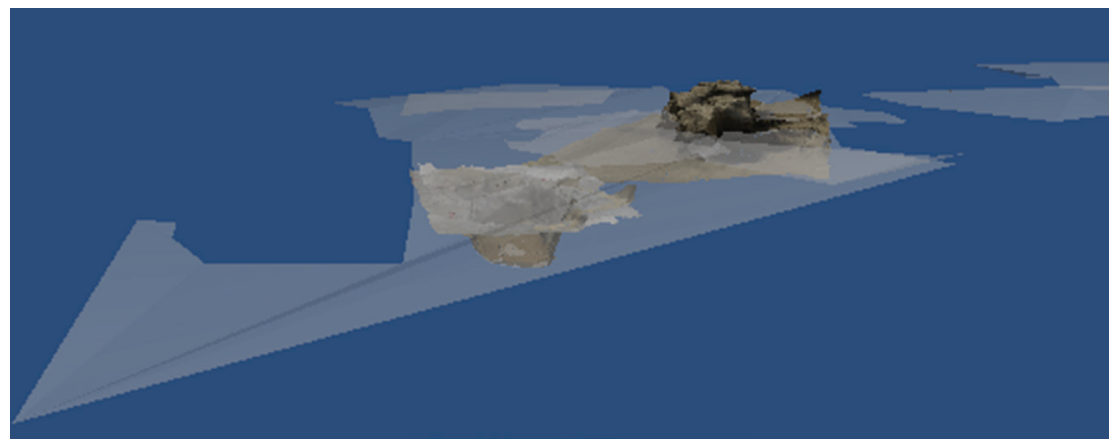

Figure 6: A screenshot from the scene assembled to investigate Marcello's question 